\begin{tabular}{|c|l|}
\hline Title & Development of a radiation detector made of a cubic boron nitride polycrystal \\
\hline Author(s) & Kaneko, J.H.; Taniguchi, T.; Kawamura, S.; Satou, K.; Fujita, F.; Homma, A.; Furusaka, M. \\
\hline Citation & $\begin{array}{l}\text { Nuclear Instruments and Methods in Physics Research Section A A ccelerators Spectrometers Detectors and A ssociated } \\
\text { Equipment, 576(2-3), 417-421 } \\
\text { https://doi.org/10.1016/.nima.2007.03.016 }\end{array}$ \\
\hline Issue Date & $2007-06-21$ \\
\hline Doc URL & http://hdl.handle.net/2115/28005 \\
\hline Type & article (author version) \\
\hline File Information & NIMP576-2-3.pdf \\
\hline
\end{tabular}

Instructions for use 


\title{
Development of a radiation detector made of a cubic boron nitride polycrystal
}

\author{
J. H. Kaneko ${ }^{1 *}$, T. Taniguchi ${ }^{2}$, S. Kawamura ${ }^{1}$, K. Satou ${ }^{1}$, F. Fujita ${ }^{1}$, A. Homma ${ }^{1}$, M. Furusaka ${ }^{1}$ \\ ${ }^{1}$ Hokkaido University, N13W8, Kita-ku, Sapporo 060-8628, Japan, \\ ${ }^{2}$ National Institute for Material Science, Namiki 1-1, Tsukuba 305-0047, Japan \\ *Corresponding Author: kin@qe.eng.hokudai.ac.jp
}

\begin{abstract}
Radiation detectors were fabricated using single and polycrystalline cubic boron nitride crystals synthesized using a high-pressure and high-temperature method. Although cBN single crystals obtained using a barium BN solvent system were nearly colorless and displayed high electrical resistance, in contrast to conventional amber colored crystals, the single crystals exhibit a leakage current that renders them unsuitable for use in a detector. In contrast, a detector made of a cBN polycrystal synthesized by direct transformation had a very low leakage current of $0.2 \mathrm{pA}$ with bias voltage of $100 \mathrm{~V}$; it functioned as a radiation detector. From an experiment using alpha particles, holes traversed a longer distance than electrons. It had a fast rise time of approximately $300 \mathrm{~ns}$. The detector also showed sensitivity to neutrons. However, output signals were smaller than the expected voltage from the experiment using alpha particles, probably because of charge accumulation from the high neutron flux.
\end{abstract}

\section{Introduction}

Cubic boron nitride, $\mathrm{cBN}$, has a diamond structure comprising boron and nitrogen atoms. Cubic boron nitride has the second-highest hardness and thermal conductivity. Moreover, it differs from diamond in its physicochemical stability for iron. Consequently, $\mathrm{cBN}$ is used widely for abrasives and cutting tools. In addition, $\mathrm{cBN}$ is a semiconductor material whose band gap is $6.3 \mathrm{eV}$. Semiconducting natures of p-type and n-type are achieved by appropriate doping during crystal growth. Mechanical, optical and electrical properties of $\mathrm{cBN}$ are reported in Ref. [1]. However, applications of $\mathrm{cBN}$ are hindered by its hardness during synthesis.

It has been possible to obtain cBN single crystals [2-4] and high-purity polycrystals of several square millimeters without any binding material [5-9]; these crystals are synthesized using a high-pressure and 
high-temperature (HP/HT) method. In the present study, which is aimed at a future application of a dosimeter for boron neutron capture therapy, the present potential of $\mathrm{cBN}$ crystals for radiation detection was examined experimentally because $\mathrm{cBN}$ is a semiconductor material that contains approximately $10.8 \mathrm{wt} \%$ of ${ }^{10} \mathrm{~B}$, a neutron-charged particle converting nucleus, even in natural abundance.

Neutron capture therapy includes drug delivery and neutron irradiation. Through drug delivery, ${ }^{10} \mathrm{~B}$ is accumulated in tumors; subsequently, neutron irradiation destroys cancer cells via the ${ }^{10} \mathrm{~B}(\mathrm{n}, \alpha)^{7} \mathrm{Li}$ reaction. Neutron fluence for tumor must be in an appropriate range in this therapy; it is evaluated using a neutron radioactivation analysis with gold wires or thermoluminescent detectors $[10,11]$ after neutron irradiation. On the other hand, real time neutron flux monitoring is ideal for this therapy. Accordingly, neutron dosimeters based on a scintillating glass optical fiber [12] or a boron-loaded plastic scintillator with an optical fiber [13] were developed. Although, these dosimeters are very effective, higher spatial resolution and measurement at multi points are required.

As a trial of a boron-based compound semiconductor to radiation detection, a cBN thin film synthesized by chemical vapor deposition method was applied as a UV-radiation sensor [14]. In addition, alpha-particle measurements using boron phosphide film in thickness of $10 \mu \mathrm{m}$ grown using hetero-epitaxy on Si have been reported [15]. No reports have described the use of thick bulk cBN for radiation detection.

\section{Synthesis of cBN crystals}

Single and polycrystalline cBN were synthesized by HP/HT methods using modified belt type high-pressure apparatus of $\mathrm{FB} 30 \mathrm{H}$ and $\mathrm{FB} 60 \mathrm{H}$. The growth conditions are described in Table 1. Figure 1 shows optical pictures of synthesized cBN single crystals and polycrystals; the grid in Fig. 1 is $1 \mathrm{~mm}$.

In general, cBN single crystals obtained using a conventional solvent, such as lithium boron nitride, were amber-colored and displayed n-type semiconduction characteristics. Apparently, this amber color and semiconducting characteristics are attributable to some defects related to oxygen and/or carbon impurities, as characterized by secondary ion mass spectroscopy analysis. For this study, we used cBN crystals obtained using a barium boron nitride solvent. Results showed that the barium BN boron nitride solvent system is beneficial to reduce oxygen and/or carbon impurities in grown cBN crystals. The resultant cBN single crystals were a transparent light-gray.

Cubic BN polycrystals were synthesized via sintering of $\mathrm{cBN}$ or hexagnal boron nitride, $\mathrm{hBN}$, powders 
under high pressure without any binder. Several differences were apparent in crystals' color from light gray to transparent black; they were attributed to the different starting materials, i.e. $\mathrm{hBN}$ or $\mathrm{cBN}$, and synthesis conditions. The band gap energy of $\mathrm{cBN}$ is reported as $6.3 \mathrm{eV}$ in Ref. [4]; an ideal $\mathrm{cBN}$ should be transparent and colorless. On the other hand, all synthesized crystals had both colors, implying that energy levels existed in their forbidden band because of impurities, defects, and stoichiometric differences. Optical properties of cBN are summarized in $\operatorname{Ref}[2,3]$.

For detector fabrication, cBN single crystals of $1-2 \mathrm{~mm}^{2}$ and polycrystals of $\phi 5 \mathrm{~mm}$ were used; thicknesses of these crystals were set at $0.3 \mathrm{~mm}$ by mechanical polishing. Figure 2 shows an example of an electron microscope image of the synthesized cBN polycrystal. The cBN polycrystal grain size was approximately 0.5-2 $\mu \mathrm{m}$. These crystals were synthesized in a condition for a small grain size suitable for cutting tools with strong mechanical strength. Therefore, it was possible to obtain 10 times larger $\mathrm{cBN}$ grains by changing the synthetic conditions.

\section{Fabrication of $\mathrm{cBN}$ radiation detectors and experiment}

\subsection{Fabrication of cBN detectors}

A semiconductor radiation detector requires application of a high bias voltage for charge collection. Therefore, higher resistivity is required for a semiconductor material. The amber cBN single crystals synthesized using the lithium BN solvent had n-type characteristics, as identified by Hall-effect measurement [15]; it was impossible to apply these crystals for radiation detection because their large leakage current. In contrast, cBN single crystals synthesized using the barium BN solvent and cBN polycrystals had higher resistivity; it was impossible to discern p-types or n-types through Hall-effect measurement up to $700^{\circ} \mathrm{C}$.

Aluminum electrodes were fabricated by evaporation after degreasing, acid processing, and rinsing with ultrapure water. No reliable information exists about suitable electrodes for $\mathrm{cBN}$; consequently, aluminum was adopted because of its ease in handling. Figure 3 shows an outer view of an example of fabricated detectors.

\subsection{I-V characteristic measurement}

For the fabricated cBN detectors, I-V characteristics were measured using a source measuring unit (237; Keithley Instruments Inc.). The detectors, which were made of the cBN single crystals, had leakage current on the order of microamperes with a bias voltage of $10 \mathrm{~V}$. For that reason, they did not function as radiation 
detectors. The detectors made of cBN polycrystals, however, had leakage current of $0.2 \mathrm{pA}$ with reverse bias voltage of $100 \mathrm{~V}$; that was a sufficiently high bias voltage and small leakage current to allow its operation as a radiation detector. Although a straight $\mathrm{I}-\mathrm{V}$ characteristic was expected because the sample had both aluminum electrodes, a strong rectification characteristic was observed. No difference existed in the surface of the cBN polycrystal on a macroscopic scale: the reason for this rectification characteristic is unclear.

\subsection{Experimental setup for radiation measurement}

For alpha particles and cold neutron measurement, a charge sensitive preamplifier (142A; Ortec), a spectroscopy amplifier (2025; Canberra) and a multi-channel analyzer (MCA/AT; Laboratory Equipment Corp. ) were used. Alpha particles from an ${ }^{241} \mathrm{Am}$ source were used in vacuum at room temperature. In addition, neutron measurements were carried out using the ultra small angle scattering (ULS) device of the University of Tokyo at the Japan Resarch Reactor 3M (JRR-3M) of the Japan Atomic Energy Agency; cold neutrons of 0.46 $\mathrm{nm}$ wavelength were used for measurement.

\section{Experimental results and discussion}

\subsection{Alpha particle measurement experiment}

Figure 4 shows examples of output signals from a preamplifier connected with the cBN polycrystal detector. The baseline of one signal is shifted upper direction in $3 \mathrm{mV}$ for ease of viewing. For all alpha particles and neutrons measurements in section 4.1 and 4.2, positively bias voltage was applied to the front-side contact, because the detector had a strong rectification characteristic as described in section 3.2. One signal was obtained in a condition where alpha particles were injected into through the front-side contact. For this condition, the main part of the output signal was created by motion of the holes because the range of the 5.5 $\mathrm{MeV}$ alpha particles in $\mathrm{cBN}$ was ca. $15 \mu \mathrm{m}$. The rectification property in a semiconductor radiation detector sometimes indicates the existence of a depletion layer. However, signals were observed in the case of bombardment of alpha particles through the back-side contact. Consequently, the detector was confirmed to work with a full depletion layer similar to an ionization chamber [16]. The cBN polycrystal detector worked with a counting rate of several tens of counts per second in a vacuum environment for alpha particles.

A rapid rise of $300 \mathrm{~ns}$ with bias voltage of $+400 \mathrm{~V}$ was observed for holes. In contrast, a slow rise of several microseconds was observed for electrons. Two reasons might explain this difference. The first reason is the 
gradient of an electric field in the crystal. The second reason is a trapping and detrapping effect on electrons, as with the HP/HT type IIa diamond radiation detector for holes [17]. Further investigation using several cBN polycrystal detectors is necessary.

Figure 5 shows pulse height spectra obtained with the cBN polycrystal detector. Even if each grain is an ideal cBN single crystal, grain boundaries were apparent at which charge trapping occurred. Consequently, no peak was apparent in the spectrum. Although lower bias voltage was applied for the condition in which the main part of output signals created by motion of holes, channels with greater pulse height than the case of electrons were observed. This result indicates that holes were moved a longer distance than electrons in the cBN polycrystal. Investigation of charge-trapping levels is indispensable to interpret this result.

\subsection{Neutron measurement experiment}

Cold neutrons were transported by a neutron guide tube from a liquid hydrogen moderator settled in the core of the JRR-3M. The cold neutrons were diffracted using pyrolytic graphite; neutron of $0.46 \mathrm{~nm}$ wavelength was sent to the ULS device. Neutron flux was approximately $1 \times 10^{6}$ at the detector position. Measurement was carried out in atmosphere at room temperature. A penetration length of $\mathrm{cBN}$ for thermal neutrons is $0.152 \mathrm{~mm}$. Therefore, all neutrons entered in the detector made some reactions, because lower energy neutrons were used in this measurement. Figure 6 shows an example of output signals obtained using the cBN polycrystal detector for neutrons. Neutrons produce a reaction with ${ }^{10} \mathrm{~B}$; then ${ }^{7} \mathrm{Li}$ of $2.78 \mathrm{MeV}$ and ${ }^{4} \mathrm{He}$ of $1.78 \mathrm{MeV}$ are created. Therefore, a pulse height in several millivolts was expected in this measurement. Although the detector was sensitive to the neutrons, only output signals smaller than $2 \mathrm{mV}$ were observed. This result was probably the result of a higher flux of neutrons than with alpha particles; it caused a polarization phenomenon in the cBN polycrystal detector. Improvement in crystal quality and investigation of charge trapping centers are indispensable for realization of a practically useful cBN neutron detector.

This research was partly supported by KAKENHI (15360498) and a research grant from the Association for Nuclear Technology in Medicine.

\section{References}

[1] O. Mishima, K. Era, “21. Science and Technology of Boron Nitride”, Electric Refractory Materials, Ed. Y. Kumashiro (Marcel Dekker Inc., 2000) 495. 
[2] T. Taniguchi, S. Yamaoka, J. Cryst. Growth, 222, (2001) 549.

[3] T. Taniguchi, K. Watanabe, S. Koizumi, Phys. Stat. Sol. A, 201, (2004) 2573.

[4] K.Watanabe, T. Taniguchi, H. Kanda, Phys. Stat. Sol. A, 201, (2004) 2561.

[5] M. Akaishi, T. Satoh, M. Ishii, T. Taniguchi, S. Yamaoka, J. Mater. Sci. Let., 12, (1993) 1883.

[6] T. Taniguchi, M. Akaishi, S. Yamaoka, J. Am. Ceram. Soc., 79(2), (1996) 547.

[7] T. Taniguchi, M. Akaishi, S. Yamaoka, J. Mater. Res., 14(1), (1999) 162.

[8] H. Sumiya, S. Uesaka, J. Mater. Sci., 35, (2000) 1181.

[9] M.P. D’Evelyn, T. Taniguchi, Diamond and Relat. Mater., 8, (1999) 1522.

[10] T. Toivonen, V. Chernov, H. Jungner, C. Aschan, A. Toivonen, Radiation Measurements, Vol. 29(3-4), (1998) 373.

[11] P. Bilski, M. Budzanowski, E. Ochab, P. Olko, L. Czopyk, Radiation Protection Sosimetry, Vol. 110, (2004) 623.

[12] M. Bliss, R. A. Craig, P. L. Reeder, D. S. Sunberg, IEEE Trans. Nuclear Science, Vol. 42(4), (1995) 639.

[13] M. Ishikawa, K. Ono, Y. Sakurai, H. Unesaki, A. Uritani, G. Bengua, T. Kobayashi, K. Tanaka, T. Kosako, Applied Radiation and Isotopes, 61, (2004) 775.

[14] J. C. Lund, F. Olschner, F. Ahmed, K. S. Shah, Proc. Mat. Res. Soc. Symp., 162, (1990)601.

[15] T. Taniguchi T. Teraji, S. Koizumi, K. Watanabe, S. Yamaoka, Jpn. J. Appl. Phys., 41, (2002) L109.

[16] G. F. Knoll, Radiation Detection and Measurement, $3^{\text {rd }}$ edition (Wiley, New York, 1999) 149.

[17] J. Kaneko, M. Katagiri, Nucl. Instrum. Meth., A383, (1996) 547. 
Table 1 Synthetic conditions and methods of cBN crystals

\begin{tabular}{|l|l|c|c|l|}
\hline & Synthesis Method & Catalyst & Capsule & $\begin{array}{l}\text { Temperature and } \\
\text { Pressure }\end{array}$ \\
\hline cBN single crystal & Temperature divergence method & $\mathrm{Ba} \mathrm{BN}$ & $\mathrm{Mo}$ & $1600^{\circ} \mathrm{C}, 5.5 \mathrm{GPa}$ \\
\hline cBN polycrystal & Direct conversion method & none & $\mathrm{Ta}$ & $2000^{\circ} \mathrm{C}, 7.7 \mathrm{GPa}$ \\
\hline
\end{tabular}




\section{Figure captions}

Figure 1 Optical photographs of a) a cBN single crystal synthesized using a barium BN solvent, b) a cBN polycrystal synthesized by direct transformation without any binders. The grid size is $1 \mathrm{~mm}$.

Figure 2 An exemplary electron microscope image of a cBN polycrystal. Grains were ca. $0.5-2.0 \mu \mathrm{m}$.

Figure 3 Exterior view of a fabricated $\mathrm{cBN}$ radiation detector. A cBN crystal was fixed by epoxy resin on an alumina base with a hole; then aluminum electrodes were fabricated for both sides by evaporation. Charged particles were injected into the detector through both sides of the electrodes.

Figure 4 Examples of output signals from a charge preamplifier (142A; Ortec) connected to a cBN polycrystal detector. The baseline of one pulse, i.e. electron, was shifted upward in $3 \mathrm{mV}$ for convenience. These signals were obtained by changing electrodes where the alpha particle was injected: major parts of these signals were created by motion of holes or electrons.

Figure 5 An example of pulse height spectra obtained using the $\mathrm{cBN}$ polycrystal detector for alpha particles. The counting rate of several tens of counts per second was achieved in a vacuum environment, but no peak was observed because a charge loss existed in the crystal.

Figure 6 Exemplary output signals from the cBN polycrystal detector for cold neutrons. The detector had response to neutrons. However, the pulse height was less than $2 \mathrm{mV}$, probably because of charge accumulation resulting from the high neutron flux of $10^{6}$. 
a)

b)

Fig.1 


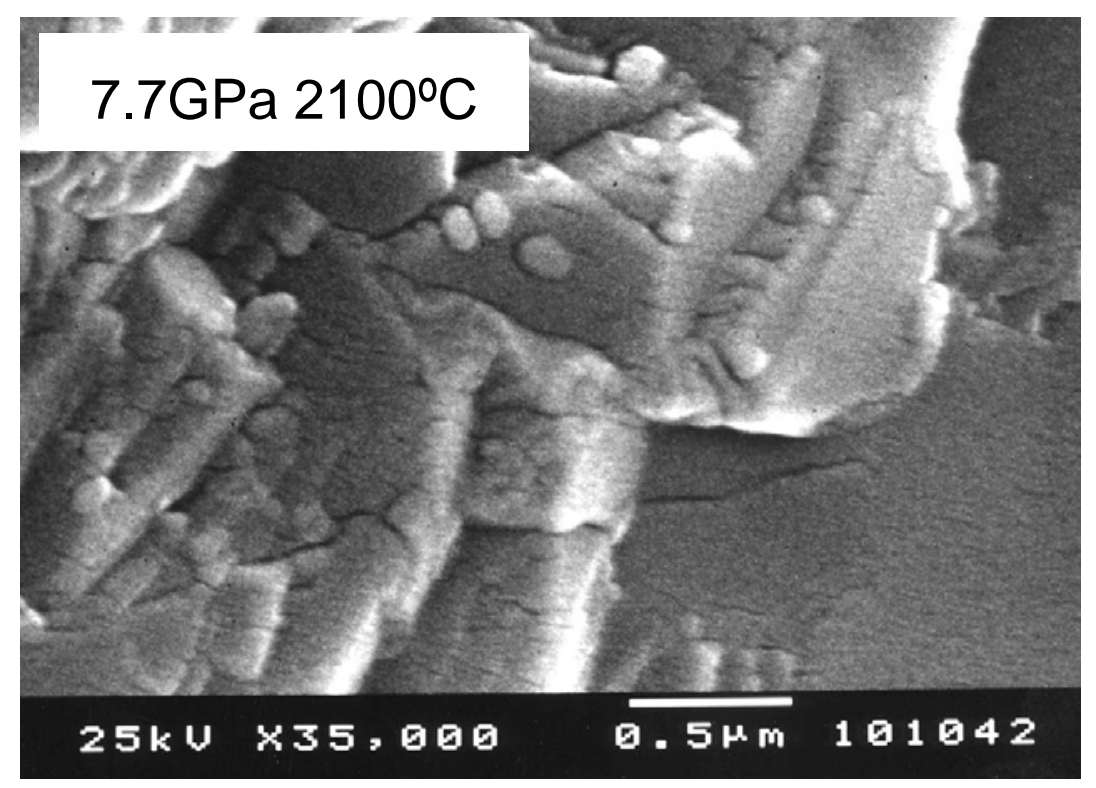

Fig.2 


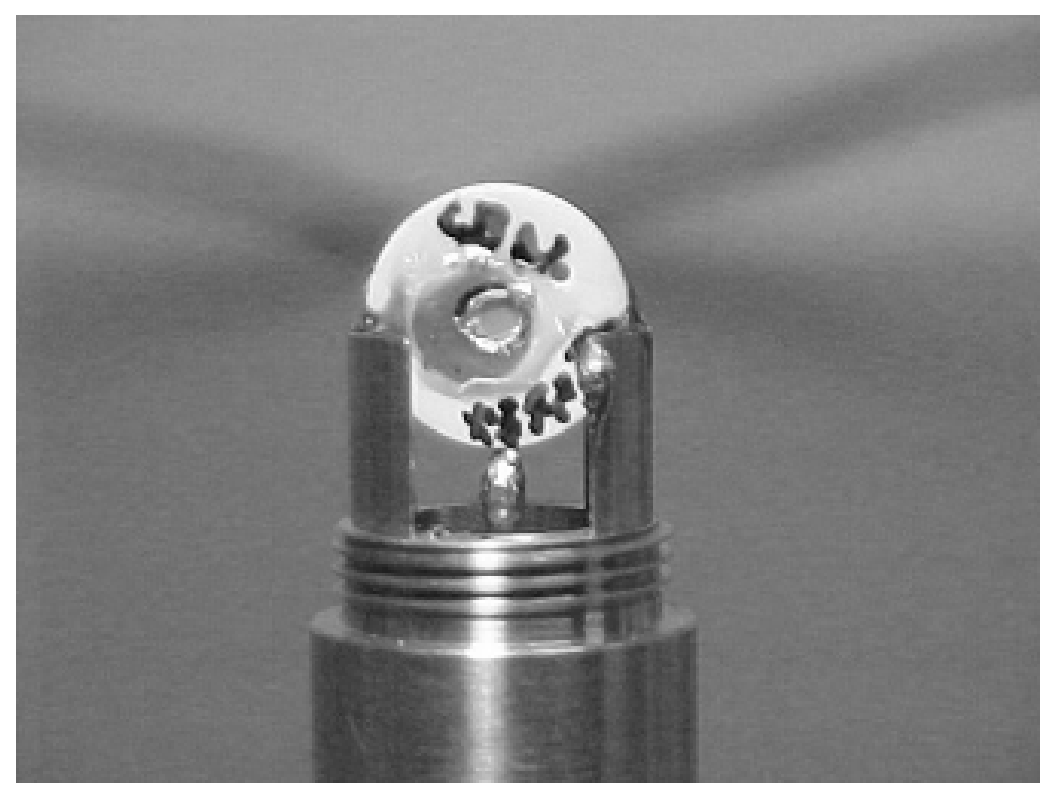

Fig. 3 


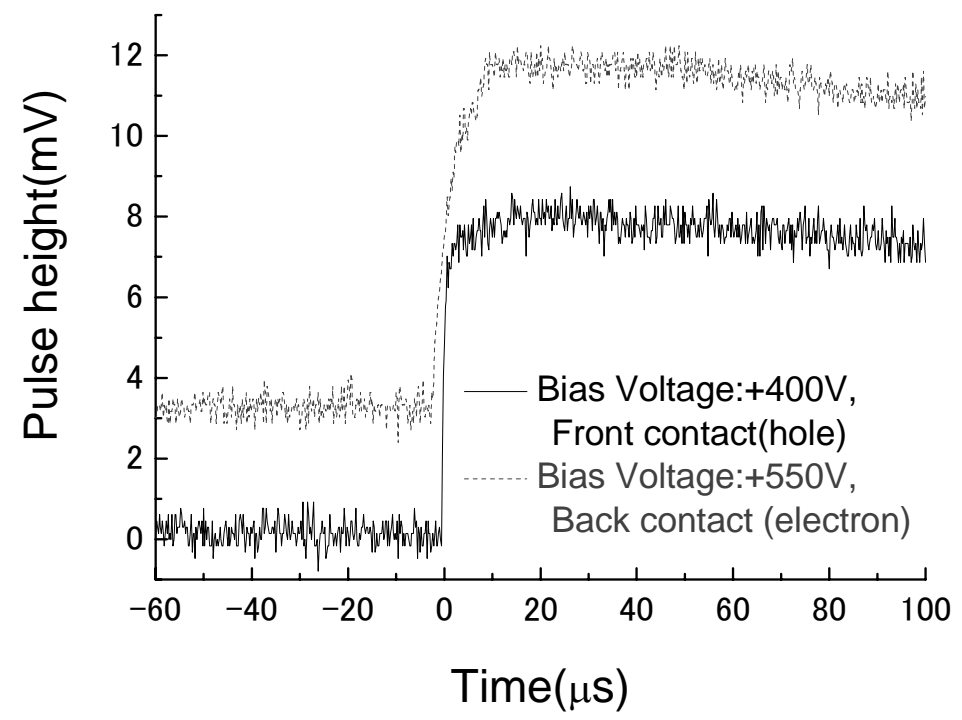

Fig.4 


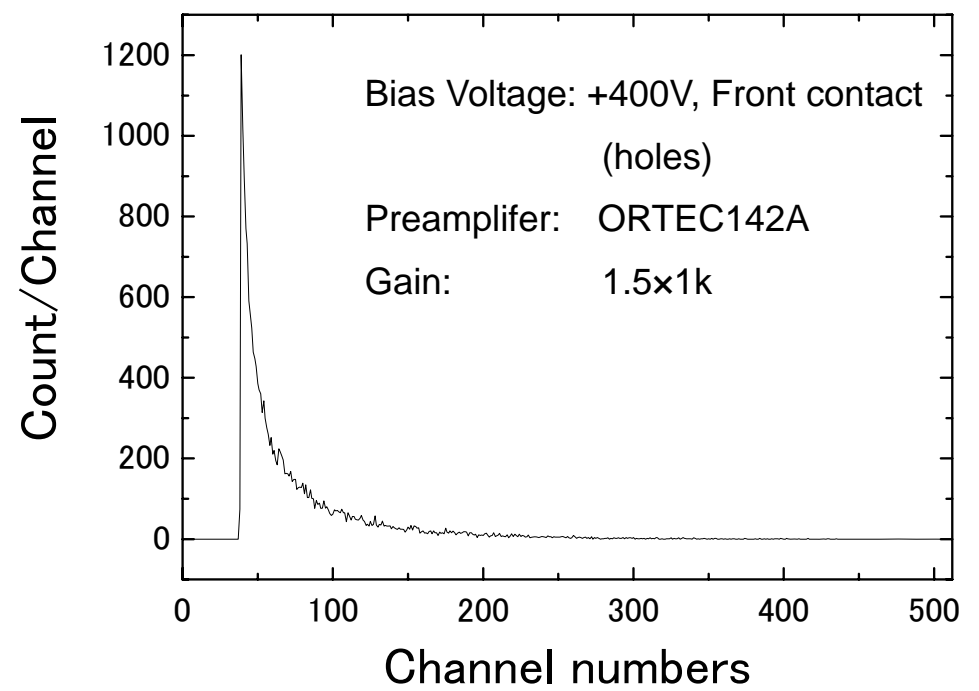

Fig.5 


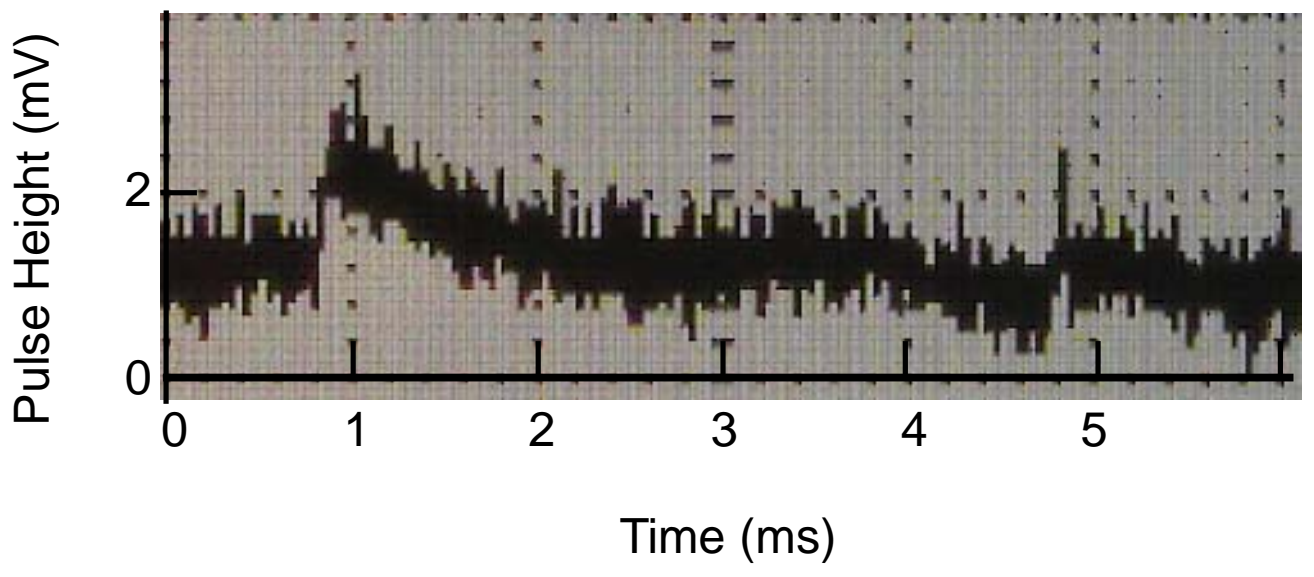

Fig. 6 